J. Cosmol. Astropart. Phys. JCAP03(2011)001

\title{
Charged fermions tunneling from accelerating and rotating black holes
}

\author{
Mudassar Rehman and K. Saifullah \\ Department of Mathematics, Quaid-i-Azam University, Islamabad, Pakistan \\ Electronic address: saifullah@qau.edu.pk
}

\begin{abstract}
We study Hawking radiation of charged fermions from accelerating and rotating black holes with electric and magnetic charges. We calculate the tunneling probabilities of incoming and outgoing fermionic particles and find the Hawking temperature of these black holes. We also provide an explicit expression of the classical action for the massive and massless particles in the background of these black holes.
\end{abstract}




\section{Introduction}

Recently there has been a lot of interest in studying the Hawking radiation [1] as a phenomenon of quantum tunneling from black holes $[2,3,4]$. In one approach the tunneling rate of particles coming out from the black hole horizon is calculated from the imaginary part of their classical action $[5,6]$. Following this approach radiation of scalar particles, and charged and uncharged fermions was studied for the Kerr, Kerr-Newman, Taub-NUT, Gödel, dilatonic black holes and those with acceleration and rotation $[7,8,9,10,11,12,13,14,15,16,17]$.

In this paper we study tunneling of charged fermions from accelerating and rotating black holes with electric and magnetic charges [18, 19, 20, 21, 22, 23]. These black holes have acceleration horizons also in addition to two rotation horizons. We find the tunneling probabilities of these particles using the WKB approximation and then calculate temperature at the horizons. The paper is organized as follows. In Section 2 we describe the spacetime for accelerating and rotating black holes with electric and magnetic charges and their basic properties. Section 3 deals with the solution of charged Dirac equation in the background of these black holes and tunneling temperature is calculated. In Section 4 we provide the exact form of the action for massless as well as massive particles. We conclude briefly in Section 5 .

\section{Accelerating and rotating black holes with electric and magnetic charges}

The Plebański and Demiański metric [19, 20, 24] covers a large family of spacetimes which include the well-known black hole solutions like Schwarzschild, ReissnerNordström, Kerr, Kerr-Newman, Kerr-NUT and many others. This family includes, in particular, solutions for accelerating and rotating black holes also with electromagnetic field, which in spherical polar coordinates can be written as

$$
\begin{aligned}
d s^{2}= & -\frac{1}{\Omega^{2}}\left[\frac{Q}{\rho^{2}} d t^{2}+\frac{Q a^{2} \sin ^{4} \theta}{\rho^{2}} d \phi^{2}-\frac{Q}{\rho^{2}} 2 a \sin ^{2} \theta d t d \phi-\frac{\rho^{2}}{Q} d r^{2}-\frac{\rho^{2}}{P} d \theta^{2}\right. \\
& \left.-\frac{P \sin ^{2} \theta}{\rho^{2}} a^{2} d t^{2}-\frac{P \sin ^{2} \theta}{\rho^{2}}\left(r^{2}+a^{2}\right)^{2} d \phi^{2}+\frac{P \sin ^{2} \theta}{\rho^{2}} 2 a\left(r^{2}+a^{2}\right) d t d \phi\right],
\end{aligned}
$$

where

$$
\Omega=1-\alpha r \cos \theta
$$




$$
\begin{aligned}
\rho^{2} & =r^{2}+a^{2} \cos ^{2} \theta, \\
P & =1-2 \alpha M \cos \theta+\left[\alpha^{2}\left(a^{2}+e^{2}+g^{2}\right)\right] \cos ^{2} \theta, \\
Q & =\left[\left(a^{2}+e^{2}+g^{2}\right)-2 M r+r^{2}\right]\left(1-\alpha^{2} r^{2}\right) .
\end{aligned}
$$

Here $M$ is the mass of the black hole, $e$ and $g$ are its electric and magnetic charges, $a$ is angular momentum per unit mass, and $\alpha$ is the acceleration of the black hole. We write the metric (2.1) in a more convenient form as

$$
\begin{aligned}
d s^{2}= & -\left(\frac{Q-a^{2} P \sin ^{2} \theta}{\rho^{2} \Omega^{2}}\right) d t^{2}+\left(\frac{\rho^{2}}{Q \Omega^{2}}\right) d r^{2}+\left(\frac{\rho^{2}}{P \Omega^{2}}\right) d \theta^{2} \\
& +\left(\frac{\sin ^{2} \theta\left[P\left(r^{2}+a^{2}\right)^{2}-a^{2} Q \sin ^{2} \theta\right]}{\rho^{2} \Omega^{2}}\right) d \phi^{2} \\
& -\left(\frac{2 a \sin ^{2} \theta\left[P\left(r^{2}+a^{2}\right)-Q\right]}{\rho^{2} \Omega^{2}}\right) d t d \phi .
\end{aligned}
$$

Now we use the notation of Ref. [8] and write the above metric as

$$
d s^{2}=-f(r, \theta) d t^{2}+\frac{d r^{2}}{g(r, \theta)}+\Sigma(r, \theta) d \theta^{2}+K(r, \theta) d \phi^{2}-2 H(r, \theta) d t d \phi,
$$

where the new functions are

$$
\begin{aligned}
f(r, \theta) & =\left(\frac{Q-a^{2} P \sin ^{2} \theta}{\rho^{2} \Omega^{2}}\right) \\
g(r, \theta) & =\frac{Q \Omega^{2}}{\rho^{2}} \\
\Sigma(r, \theta) & =\left(\frac{\rho^{2}}{P \Omega^{2}}\right) \\
K(r, \theta) & =\left(\frac{\sin ^{2} \theta\left[P\left(r^{2}+a^{2}\right)^{2}-a^{2} Q \sin ^{2} \theta\right]}{\rho^{2} \Omega^{2}}\right) \\
H(r, \theta) & =\left(\frac{a \sin ^{2} \theta\left[P\left(r^{2}+a^{2}\right)-Q\right]}{\rho^{2} \Omega^{2}}\right)
\end{aligned}
$$

The electromagnetic vector potential for these black holes is given by [20]

$$
A=\frac{-e r\left[d t-a \sin ^{2} \theta d \phi\right]-g \cos \theta\left[a d t-\left(r^{2}+a^{2}\right) d \phi\right]}{r^{2}+a^{2} \cos ^{2} \theta} .
$$


The event horizons of these black holes are obtained by putting $g(r, \theta)=0$, which gives their location at

$$
r=\frac{1}{\alpha \cos \theta}, r= \pm \frac{1}{\alpha}, \text { and } r_{ \pm}=M \pm \sqrt{M^{2}-a^{2}-e^{2}-g^{2}}
$$

where $r_{ \pm}$represent the outer and inner horizons corresponding to the Kerr-Newman black holes. The other horizons are acceleration horizons. In this paper we study only the non-extremal black holes i.e. those cases where the quantity under the radical sign in Eq. (2.14) remains positive. The angular velocity at the black hole horizon $[8,17]$

$$
\Omega_{H}=\frac{H\left(r_{+}, \theta\right)}{K\left(r_{+}, \theta\right)}
$$

becomes

$$
\Omega_{H}=\frac{a}{\left(r_{+}^{2}+a^{2}\right)}
$$

We define another function which will be needed later

$$
F(r, \theta)=f(r, \theta)+\frac{H^{2}(r, \theta)}{K(r, \theta)} .
$$

Using values of $f, K$ and $H$ this takes the form

$$
F(r, \theta)=\frac{P Q \rho^{2}}{\Omega^{2}\left[P\left(r^{2}+a^{2}\right)^{2}-a^{2} Q \sin ^{2} \theta\right]} .
$$

\section{Tunneling of charged fermions}

For studying tunneling of charged fermions, we will solve the Dirac equation for accelerating and rotating black holes with electric and magnetic charges. The covariant Dirac equation with electric charge $q$ is

$$
i \gamma^{\mu}\left(D_{\mu}-\frac{i q}{\hbar} A_{\mu}\right) \Psi+\frac{m}{\hbar} \Psi=0
$$

where $m$ is the mass of the fermion particles and $D_{\mu}=\partial_{\mu}+\Omega_{\mu}$, with $\Omega_{\mu}=\frac{1}{2} i \Gamma_{\mu}^{\alpha \beta} \Sigma_{\alpha \beta}$, and $\Sigma_{\alpha \beta}=\frac{1}{4} i\left[\gamma^{\alpha}, \gamma^{\beta}\right]$ is antisymmetric. The quantities $\gamma^{\prime} s$ are defined as

$$
\begin{aligned}
\gamma^{t} & =\frac{1}{\sqrt{F(r, \theta)}} \gamma^{0}, \gamma^{r}=\sqrt{g(r, \theta)} \gamma^{3}, \gamma^{\theta}=\frac{1}{\sqrt{\sum(r, \theta)}} \gamma^{1}, \\
\gamma^{\phi} & =\frac{1}{\sqrt{K(r, \theta)}}\left(\gamma^{2}+\frac{H(r, \theta)}{\sqrt{F(r, \theta) K(r, \theta)}} \gamma^{0}\right),
\end{aligned}
$$


where the matrices $\gamma^{\mu}(\mu=0,1,2,3)$ are

$$
\begin{aligned}
& \gamma^{0}=\left(\begin{array}{cc}
0 & I \\
-I & 0
\end{array}\right), \gamma^{1}=\left(\begin{array}{cc}
0 & \sigma^{1} \\
\sigma^{1} & 0
\end{array}\right), \\
& \gamma^{2}=\left(\begin{array}{cc}
0 & \sigma^{2} \\
\sigma^{2} & 0
\end{array}\right), \gamma^{3}=\left(\begin{array}{cc}
0 & \sigma^{3} \\
\sigma^{3} & 0
\end{array}\right) .
\end{aligned}
$$

The Pauli matrices $\sigma^{i}(i=1,2,3)$ are

$$
\sigma^{1}=\left(\begin{array}{ll}
0 & 1 \\
1 & 0
\end{array}\right) \sigma^{2}=\left(\begin{array}{cc}
0 & -i \\
i & 0
\end{array}\right), \sigma^{3}=\left(\begin{array}{cc}
1 & 0 \\
0 & -1
\end{array}\right)
$$

Note that

$$
\gamma^{5}=i \gamma^{t} \gamma^{r} \gamma^{\theta} \gamma^{\phi}=\sqrt{\frac{g}{F K \Sigma}}\left(\begin{array}{cc}
-I+\frac{H}{\sqrt{F K}} \sigma^{2} & 0 \\
0 & I+\frac{H}{\sqrt{F K}} \sigma^{2}
\end{array}\right),
$$

is the resulting $\gamma^{5}$ matrix. The eigenvector of $\sigma^{3}$ for the spin up and spin down cases are $\xi_{\uparrow}=\left(\begin{array}{l}1 \\ 0\end{array}\right)$ and $\xi_{\downarrow}=\left(\begin{array}{l}0 \\ 1\end{array}\right)$. We take the following assumptions for the solution of the spin up and spin down particles respectively

$$
\begin{aligned}
& \Psi_{\uparrow}(t, r, \theta, \phi)=\left(\begin{array}{l}
A(t, r, \theta, \phi) \xi_{\uparrow} \\
B(t, r, \theta, \phi) \xi_{\uparrow}
\end{array}\right) \exp \left[\frac{i}{\hbar} I_{\uparrow}(t, r, \theta, \phi)\right], \\
& \Psi_{\downarrow}(t, r, \theta, \phi)=\left(\begin{array}{l}
A(t, r, \theta, \phi) \xi_{\downarrow} \\
B(t, r, \theta, \phi) \xi_{\downarrow}
\end{array}\right) \exp \left[\frac{i}{\hbar} I_{\downarrow}(t, r, \theta, \phi)\right],
\end{aligned}
$$

where $I_{\uparrow / \downarrow}$ denote the action of the emitted spin up and spin down particles, respectively. We will only give calculations for the spin up case; those for the spin down case are similar, apart from the change in sign. Now using the antisymmetric property i.e. $\left[\gamma^{\alpha}, \gamma^{\beta}\right]=0$, if $\alpha=\beta$ and $\left[\gamma^{\alpha}, \gamma^{\beta}\right]=-\left[\gamma^{\beta}, \gamma^{\alpha}\right]$, if $\alpha \neq \beta$, we find that the Dirac Eq. (3.1) reduces to

$$
i \gamma^{\mu}\left(\partial_{\mu}-\frac{i q}{\hbar} A_{\mu}\right) \Psi+\frac{m}{\hbar} \Psi=0
$$

Substituting the ansatz (3.3) and (3.4) into the Dirac equation we obtain after some algebra the following four equations 


$$
\begin{gathered}
0=-B\left[\frac{1}{\sqrt{F(r, \theta)}} \partial_{t} I_{\uparrow}+\sqrt{g(r, \theta)} \partial_{r} I_{\uparrow}+\frac{H(r, \theta)}{K(r, \theta) \sqrt{F(r, \theta)}} \partial_{\phi} I_{\uparrow}\right. \\
\left.-\frac{1}{\sqrt{F(r, \theta)}} q A_{t}-\frac{H(r, \theta)}{K(r, \theta) \sqrt{F(r, \theta)}} q A_{\phi}\right]+m A, \\
0=-B\left[\frac{1}{\sqrt{\Sigma(r, \theta)}} \partial_{\theta} I_{\uparrow}+\frac{i}{\sqrt{K(r, \theta)}} \partial_{\phi} I_{\uparrow}-\frac{i}{\sqrt{K(r, \theta)}} q A_{\phi}\right], \\
0=A\left[\frac{1}{\sqrt{F(r, \theta)}} \partial_{t} I_{\uparrow}-\sqrt{g(r, \theta)} \partial_{r} I_{\uparrow}+\frac{H(r, \theta)}{K(r, \theta) \sqrt{F(r, \theta)}} \partial_{\phi} I_{\uparrow}\right. \\
0=-A\left[\frac{1}{\sqrt{F(r, \theta)}} q A_{t}-\frac{H(r, \theta)}{K(r, \theta) \sqrt{F(r, \theta)}} q A_{\phi}\right]+m B, \\
{\left[\frac{1}{\sqrt{\Sigma(r, \theta)}} \partial_{\theta} I_{\uparrow}+\frac{i}{\sqrt{K(r, \theta)}} \partial_{\phi} I_{\uparrow}-\frac{i}{\sqrt{K(r, \theta)}} q A_{\phi}\right] .}
\end{gathered}
$$

Now the metric coefficients do not depend on $t$ and $\phi$ coordinates therefore we can apply the standard ansatz $[8,9,17]$

$$
I_{\uparrow}=-E t+J \phi+W(r, \theta),
$$

where $E$ and $J$ denote the energy and angular momentum of the emitted particle. Now, expanding $g(r, \theta)$ and $F(r, \theta)$ using Taylor's theorem near the outer horizon and neglecting squares and higher powers, as has been done in the case of uncharged particles and neutral black holes [17], and substituting the values of $A_{t}\left(r_{+}, \theta\right), A_{\phi}\left(r_{+}, \theta\right), \Sigma\left(r_{+}, \theta\right)$ and $K\left(r_{+}, \theta\right)$ the above set of equations take the form

$$
\begin{gathered}
-B\left[\frac{\left(-E+\Omega_{H} J+\frac{q e r_{+}}{\left(r_{+}^{2}+a^{2}\right)}\right)}{\sqrt{\left(r-r_{+}\right) \partial_{r} F\left(r_{+}, \theta\right)}}+\sqrt{\left(r-r_{+}\right) \partial_{r} g\left(r_{+}, \theta\right)}\left(\partial_{r} W\right)\right]+m A=0 \\
-B\left[\sqrt{\frac{P \Omega^{2}\left(r_{+}, \theta\right)}{\rho^{2}\left(r_{+}, \theta\right)} \partial_{\theta} W}\right. \\
\left.+\frac{i \rho\left(r_{+}, \theta\right) \Omega\left(r_{+}, \theta\right)}{\sqrt{\sin ^{2} \theta P\left(r_{+}^{2}+a^{2}\right)^{2}}}\left(J-q\left(\frac{a e r_{+} \sin ^{2} \theta+g\left(r_{+}^{2}+a^{2}\right) \cos \theta}{r_{+}^{2}+a^{2} \cos ^{2} \theta}\right)\right)\right]=0
\end{gathered}
$$




$$
\begin{aligned}
& A\left[\frac{\left(-E+\Omega_{H} J+\frac{q e r_{+}}{\left(r_{+}^{2}+a^{2}\right)}\right)}{\sqrt{\left(r-r_{+}\right) \partial_{r} F\left(r_{+}, \theta\right)}}-\sqrt{\left(r-r_{+}\right) \partial_{r} g\left(r_{+}, \theta\right)} \partial_{r} W\right]+B m=0 . \\
& -A\left[\sqrt{\frac{P \Omega^{2}\left(r_{+}, \theta\right)}{\rho^{2}\left(r_{+}, \theta\right)} \partial_{\theta} W}\right. \\
& \left.+\frac{i \rho\left(r_{+}, \theta\right) \Omega\left(r_{+}, \theta\right)}{\sqrt{\sin ^{2} \theta P\left(r_{+}^{2}+a^{2}\right)^{2}}}\left(J-q\left(\frac{a e r_{+} \sin ^{2} \theta+g\left(r_{+}^{2}+a^{2}\right) \cos \theta}{r_{+}^{2}+a^{2} \cos ^{2} \theta}\right)\right)\right]=0 .
\end{aligned}
$$

It is possible to sperate $W(r, \theta)$ near the black hole horizon as

$$
W(r, \theta)=R(r)+\Theta(\theta) .
$$

First we solve Eqs. (3.11) - (3.14) for the massless case i.e. $m=0$. Using the above separation and taking $m=0$ we get

$$
-B\left[\frac{\left(-E+\Omega_{H} J+\frac{q e r_{+}}{\left(r_{+}^{2}+a^{2}\right)}\right)}{\sqrt{\left(r-r_{+}\right) \partial_{r} F\left(r_{+}, \theta\right)}}+\sqrt{\left(r-r_{+}\right) \partial_{r} g\left(r_{+}, \theta\right)} R^{\prime}(r)\right]=0,
$$

which implies

$$
R^{\prime}(r)=R_{+}^{\prime}(r)=\frac{\left(E-\Omega_{H} J-\frac{q e r_{+}}{\left(r_{+}^{2}+a^{2}\right)}\right)\left(r_{+}^{2}+a^{2}\right)}{2\left(r-r_{+}\right)\left(r_{+}-M\right)\left(1-\alpha^{2} r_{+}^{2}\right)},
$$

where $R_{+}$corresponds to the outgoing solution. Similarly the incoming solution is obtained as

$$
R^{\prime}(r)=R_{-}^{\prime}(r)=-\frac{\left(E-\Omega_{H} J-\frac{q e r_{+}}{\left(r_{+}^{2}+a^{2}\right)}\right)\left(r_{+}^{2}+a^{2}\right)}{2\left(r-r_{+}\right)\left(r_{+}-M\right)\left(1-\alpha^{2} r_{+}^{2}\right)} .
$$

The imaginary part of $R_{+}$is

$$
I m R_{+}=\frac{\pi}{2} \frac{\left(E-\Omega_{H} J-\frac{q e r_{+}}{\left(r_{+}^{2}+a^{2}\right)}\right)\left(r_{+}^{2}+a^{2}\right)}{\left(r_{+}-M\right)\left(1-\alpha^{2} r_{+}^{2}\right)} .
$$


Similarly

$$
I m R_{-}=\frac{-\pi\left(E-\Omega_{H} J-\frac{q e r_{+}}{\left(r_{+}^{2}+a^{2}\right)}\right)\left(r_{+}^{2}+a^{2}\right)}{2\left(r_{+}-M\right)\left(1-\alpha^{2} r_{+}^{2}\right)}
$$

showing that

$$
\operatorname{Im} R_{+}=-\operatorname{Im} R_{-} .
$$

The probabilities of crossing the horizon in each direction are proportional to $[5,6]$

$$
\begin{aligned}
& P_{\text {emission }} \propto \exp [-2 \operatorname{ImI}]=\exp \left[-2\left(\operatorname{Im} R_{+}+\operatorname{Im} \Theta\right)\right] \\
& P_{\text {absorption }} \propto \exp [-2 \operatorname{Im} I]=\exp \left[-2\left(\operatorname{Im} R_{-}+\operatorname{Im} \Theta\right)\right] .
\end{aligned}
$$

Thus the probability of a particle tunneling from inside to outside the horizon is

$$
\Gamma \propto \frac{P_{\text {emission }}}{P_{\text {absorption }}}=\frac{\exp \left[-2\left(\operatorname{Im} R_{+}+\operatorname{Im} \Theta\right)\right]}{\exp \left[-2\left(\operatorname{Im} R_{-}+\operatorname{Im} \Theta\right)\right]}
$$

or

$$
\Gamma=\exp \left[-2\left(I m R_{+}-\operatorname{Im} R_{-}\right)\right]
$$

and using Eq. (3.20)

$$
\Gamma=\exp \left[-4 I m R_{+}\right]
$$

which in view of Eq. (3.18) becomes

$$
\Gamma=\exp \left[\frac{-2 \pi\left(r_{+}^{2}+a^{2}\right)}{\left(r_{+}-M\right)\left(1-\alpha^{2} r_{+}^{2}\right)}\left(E-\Omega_{H} J-\frac{q e r_{+}}{\left(r_{+}^{2}+a^{2}\right)}\right)\right] .
$$

Comparing this with $\Gamma=\exp [-\beta E]$ where $\beta=1 / T_{H}$ we find that the Hawking temperature $[5,6]$ is given by

$$
T_{H}=\frac{\left(r_{+}-M\right)\left(1-\alpha^{2} r_{+}^{2}\right)}{2 \pi\left(r_{+}^{2}+a^{2}\right)} .
$$

Note that if we put acceleration equal to zero in formulae (3.23), we recover the tunneling probability for the Kerr-Newman black hole [7]. Similarly, putting rotation equal to zero will yield the formula for the Reissner-Nordström black hole. It is worth mentioning here that from Eqs. (3.21)-(3.23) it seems that for some value of $E$ and $J$ it is possible that the probabilities become greater than 1 and hence violate unitarity. However, this does not happen because apart from the spatial 
contribution the temporal part also contributes to the imaginary part $\operatorname{Im}(E \Delta t)$ of the action $[25,26]$. The time is shifted by some imaginary amount which contributes both to $P_{\text {emission }}$ and $P_{\text {absorption }}$ and results in a correct value of $\Gamma$. If this contribution is not taken into account, one gets the Hawking temperature twice as large as the original value, the so-called factor of 2 issue $[27,28]$. For the massive case $(m \neq 0)$ we again obtain the same temperature which is not unexpected [17], as the behaviour of massive particles near the black hole horizon is same as that of the massless.

\section{Calculation of the action}

In order to obtain the explicit expression for the action $I_{\uparrow}$ in the spin-up case, we solve Eqs. (3.11)-(3.14) near the black hole horizon. On using Eq. (3.15) we can write Eq. (3.11) in the form

$$
R^{\prime}(r)=\frac{m A}{B \sqrt{\partial_{r} g\left(r_{+}, \theta\right)\left(r-r_{+}\right)}}-\frac{\left(-E+\Omega_{H} J+\frac{q e r_{+}}{\left(r_{+}^{2}+a^{2}\right)}\right)}{\sqrt{\partial_{r} F\left(r_{+}, \theta\right) \partial_{r} g\left(r_{+}, \theta\right)}\left(r-r_{+}\right)} .
$$

Integrating with respect to $r$ we get

$R(r)=R_{+}(r)=\int \frac{m A}{B \sqrt{\partial_{r} g\left(r_{+}, \theta\right)\left(r-r_{+}\right)}} d r-\frac{\left(-E+\Omega_{H} J+\frac{q e r_{+}}{\left(r_{+}^{2}+a^{2}\right)}\right)}{\sqrt{\partial_{r} F\left(r_{+}, \theta\right) \partial_{r} g\left(r_{+}, \theta\right)}} \ln \left(r-r_{+}\right)$.

Similarly for the incoming particles we obtain from Eq. (3.13)

$R(r)=R_{-}(r)=\int \frac{m B}{A \sqrt{\partial_{r} g\left(r_{+}, \theta\right)\left(r-r_{+}\right)}} d r+\frac{\left(-E+\Omega_{H} J+\frac{q e r_{+}}{\left(r_{+}^{2}+a^{2}\right)}\right)}{\sqrt{\partial_{r} F\left(r_{+}, \theta\right) \partial_{r} g\left(r_{+}, \theta\right)}} \ln \left(r-r_{+}\right)$.

From Eq. (3.12) (or (3.14)) on using Eq. (3.15) we obtain

$$
\begin{aligned}
& \sqrt{\frac{P \Omega^{2}\left(r_{+}, \theta\right)}{\rho^{2}\left(r_{+}, \theta\right)}} \partial_{\theta} \Theta \\
+ & \frac{i \rho\left(r_{+}, \theta\right) \Omega\left(r_{+}, \theta\right)}{\sqrt{\sin ^{2} \theta\left[P\left(r_{+}^{2}+a^{2}\right)^{2}\right]}}\left(J-q\left(\frac{a e r_{+} \sin ^{2} \theta+g\left(r_{+}^{2}+a^{2}\right) \cos \theta}{r_{+}^{2}+a^{2} \cos ^{2} \theta}\right)\right)=0 .
\end{aligned}
$$


Substituting the values of $\rho$ and $P$ from Eqs. (2.3) and (2.4) and after simplifying this yields

$$
\begin{aligned}
\partial_{\theta} \Theta= & \frac{\left(\text { iqaer }_{+}+i J a^{2}\right) \sin \theta}{\left(r_{+}^{2}+a^{2}\right)\left[1-2 \alpha M \cos \theta+\alpha^{2}\left(a^{2}+e^{2}+g^{2}\right) \cos ^{2} \theta\right]} \\
& +\frac{i q g \cos \theta-i J}{\sin \theta\left[1-2 \alpha M \cos \theta+\alpha^{2}\left(a^{2}+e^{2}+g^{2}\right) \cos ^{2} \theta\right]} .
\end{aligned}
$$

Integrating with respect to $\theta$ we get

$$
\Theta=\frac{i q a e r_{+}+i J a^{2}}{r_{+}^{2}+a^{2}} I_{1}+I_{2},
$$

where $I_{1}$ and $I_{2}$ are obvious substitutions, which after evaluating the integrals come out to be

$$
\begin{gathered}
I_{1}=-\frac{1}{2 \alpha \sqrt{M^{2}-a^{2}-e^{2}-g^{2}}} \ln \left(\frac{\alpha \cos \theta\left(a^{2}+e^{2}+g^{2}\right)-M-\sqrt{M^{2}-a^{2}-e^{2}-g^{2}}}{\alpha \cos \theta\left(a^{2}+e^{2}+g^{2}\right)-M+\sqrt{M^{2}-a^{2}-e^{2}-g^{2}}}\right), \\
I_{2}=L_{1} \ln (1-\cos \theta)+L_{2} \ln (1+\cos \theta) \\
-L_{3} \ln \left[1-2 \alpha M \cos \theta+\alpha^{2}\left(a^{2}+e^{2}+g^{2}\right) \cos ^{2} \theta\right] \\
-L_{4} \ln \left[\frac{\alpha\left(a^{2}+e^{2}+g^{2}\right) \cos \theta-M-\sqrt{M^{2}-a^{2}-e^{2}-g^{2}}}{\alpha\left(a^{2}+e^{2}+g^{2}\right) \cos \theta-M+\sqrt{M^{2}-a^{2}-e^{2}-g^{2}}}\right]
\end{gathered}
$$

where $L_{i}$ are given below

$$
\begin{aligned}
L_{1} & =\frac{i q g-i J}{2\left[1-2 \alpha M+\alpha^{2}\left(a^{2}+e^{2}+g^{2}\right)\right]} \\
L_{2} & =\frac{i q g+i J}{2\left[1+2 \alpha M+\alpha^{2}\left(a^{2}+e^{2}+g^{2}\right)\right]} \\
L_{3} & =\frac{i q g\left(1+\alpha^{2}\left(a^{2}+e^{2}+g^{2}\right)\right)-2 \alpha M i J}{2\left[1-2 \alpha M+\alpha^{2}\left(a^{2}+e^{2}+g^{2}\right)\right]\left[1+2 \alpha M+\alpha^{2}\left(a^{2}+e^{2}+g^{2}\right)\right]} \\
L_{4} & =\frac{\left(a^{2}+e^{2}+g^{2}\right)\left[-i J \alpha^{2}-i J \alpha^{4}\left(a^{2}+e^{2}+g^{2}\right)+i q g \alpha^{3} M\right]+2 i J \alpha^{2} M^{2}-i q g \alpha M}{2 \alpha \sqrt{M^{2}-a^{2}-e^{2}-g^{2}}\left[\left\{1+\alpha^{2}\left(a^{2}+e^{2}+g^{2}\right)\right\}^{2}-4 \alpha^{2} M^{2}\right]} .
\end{aligned}
$$

Using these values of $I_{1}$ and $I_{2}$ in Eq. (4.4) we get $W(r, \theta)$ from Eq. (3.15) and an explicit expression for the action of the outgoing massive particles. Putting $m=0$ will give action for the massless case. Similarly, we obtain results for the incoming fermions as well. 


\section{The acceleration horizon}

As mentioned earlier, apart from the rotation horizons, these black holes admit acceleration horizons, $r_{\alpha}=1 / \alpha \cos \theta$ and $\pm 1 / \alpha$, as well. Here we calculate the tunneling probability of fermions from these horizons. Proceeding as before we evaluate angular velocity and radial derivatives of functions $g$ and $F$ at the acceleration horizons and obtain a corresponding set of four equations like Eqs. (3.11) to (3.14). An analysis similar to the one done earlier for the massive case yields for the outgoing and incoming fermions

$$
\begin{gathered}
R_{+}=\frac{\pi i\left(E-\Omega_{\alpha} J-q e r_{\alpha} /\left(r_{\alpha}^{2}+a^{2}\right)\right)\left(r_{\alpha}^{2}+a^{2}\right)}{2 \alpha^{2} r_{\alpha}\left[r_{\alpha}^{2}-2 M r_{\alpha}+\left(a^{2}+e^{2}+g^{2}\right)\right]}, \\
R_{-}=-\frac{\pi i\left(E-\Omega_{\alpha} J-q e r_{\alpha} /\left(r_{\alpha}^{2}+a^{2}\right)\right)\left(r_{\alpha}^{2}+a^{2}\right)}{2 \alpha^{2} r_{\alpha}\left[r_{\alpha}^{2}-2 M r_{\alpha}+\left(a^{2}+e^{2}+g^{2}\right)\right]} .
\end{gathered}
$$

Thus the probability of tunneling comes out to be

$$
\Gamma=\exp \left[\frac{-2 \pi\left(r_{\alpha}^{2}+a^{2}\right)\left(E-\Omega_{\alpha} J-q e r_{\alpha} /\left(r_{\alpha}^{2}+a^{2}\right)\right)}{\alpha^{2} r_{\alpha}\left[r_{\alpha}^{2}-2 M r_{\alpha}+\left(a^{2}+e^{2}+g^{2}\right)\right]}\right] .
$$

As before we compare this expression with $\Gamma=\exp [-\beta E]$ to write the Hawking temperature at the acceleration horizon as

$$
T_{H}=\frac{\alpha^{2} r_{\alpha}\left[r_{\alpha}^{2}-2 M r_{\alpha}+\left(a^{2}+e^{2}+g^{2}\right)\right]}{2 \pi\left(r_{\alpha}^{2}+a^{2}\right)} .
$$

\section{Conclusion}

Hawking radiation has been studied as a phenomenon of quantum tunneling from black hole horizons using different methods including the Newman-Penrose formalism and Hamilton-Jacobi method. We have used the semi-classical approach to study tunneling of charged fermions from accelerating and rotating black holes having electric and magnetic charges. From the tunneling probability of the incoming and outgoing particles we have worked out the Hawking temperature of these black holes. We know that classically only the outgoing particles face the barrier but in the semiclassical approach we have followed both the incoming as well as outgoing particles see the horizon as the tunneling barrier.

Secondly, we note that the tunneling probability of charged fermions from inside to outside the horizon does not depend upon the mass of the fermion but its charge. 
The Hawking temperature depends upon acceleration as well apart from the mass, rotation parameter and the electric and magnetic charges of the black hole. However, the amount of acceleration is restricted by the value of other parameters [21, 22, 23].

\section{Acknowledgments}

Some helpful comments from Douglas Singleton are gratefully acknowledged.

\section{References}

[1] S. W. Hawking, Commun. Math. Phys. 43 (1975) 199.

[2] M. K. Parikh and F. Wilczek, Phys. Rev. Lett. 85 (2000) 5042 [hep-th/9907001].

[3] P. Kraus and F. Wilczek, Nucl. Phys. B 433 (1995) 403.

[4] M. K. Parikh, Gen. Rel. Grav. 36 (2004) 2419 [hep-th/0405160].

[5] K. Srinivasan and T. Padmanabhan, Phys. Rev. D 60 (1999) 24007.

[6] S. Shankaranarayanan, T. Padmanabhan and K. Srinivasan, Class. Quant. Grav. 19 (2002) 2671.

[7] R. Kerner and R. B. Mann, Phys. Rev. D 73 (2006) 104010.

[8] R. Kerner and R. B. Mann, Class. Quant. Grav. 25 (2008) 095014.

[9] R. Kerner and R. B. Mann, Phys. Lett. B 665 (2008) 277.

[10] S. Zhou and W. Liu, Phys. Rev. D 77 (2008) 104021.

[11] R. Li, J. R. Ren and S. W. Wei, Class. Quant. Grav. 25 (2008) 125016.

[12] D. Y. Chen, Q. Q. Jiang and X. T. Zu, Class. Quant. Grav. 25 (2008) 205022.

[13] Q. Q. Jiang, Phys. Lett. B 666 (2008) 517.

[14] X. X. Zeng and S. Z. Yang, Gen. Rel. Grav. 40 (2008) 2107.

[15] C. Ding and J. Jing, Class. Quant. Grav. 27 (2010) 035004.

[16] J. Yang and S. Z. Yang, J. Geom. Phys. 60 (2010) 986.

[17] U. A. Gillani and K. Saifullah, Tunneling of Dirac particles from accelerating and rotating black holes [arXiv:1010.6106]. 
[18] J. Podolský and H. Kadlecová, Class. Quantum Grav. 26 (2009) 105007.

[19] J. B. Griffiths and J. Podolský, Class. Quantum Grav. 22 (2005) 3467.

[20] J. Podolský and J. B. Griffiths, Phys. Rev. D 73 (2006) 044018.

[21] O. J. C. Dias and J. P. S. Lemos, Phys. Rev. D 67 (2003) 064001.

[22] O. J. C. Dias and J. P. S. Lemos, Phys. Rev. D 67 (2003) 084018.

[23] M. Bilal and K. Saifullah, Thermodynamics of accelerating and rotating black holes [arXiv:1010.5575].

[24] J. F. Plebański and M. Demiański, Ann. Phys. NY 98 (1976) 98.

[25] V. Akhmedova, T. Pilling, A. de Gill and D. Singleton, Phys. Lett. B 666 (2008) 269.

[26] V. Akhmedova, T. Pilling and D. Singleton, Int. J. Mod. Phys. D 17 (2008) 2453.

[27] E. T. Akhmedov, V. Akhmedova and D. Singleton, Phys. Lett. B 642 (2006) 124.

[28] T. Pilling, Phys. Lett. B 660 (2008) 402. 